\title{
L'ATTIVITÀ LEGISLATIVA \\ DEL PARLAMENTO ITALIANO: \\ CONSOCIATIVISMO? POLARIZZAZIONE?
}

\author{
di Francesco Zucchini
}

Consociativismo: le alterne (s)fortune di un'etichetta

Pochi termini politologici hanno avuto altrettanto successo nella polemica politica italiana della parola consociativismo. Nonostante la sua origine specialistica, che non rimanda a nessuna nozione giuridica né ad alcuna dottrina politica o sociale, è difficile assistere ad un dibattito politico senza che qualcuno dei partecipanti non lanci alla parte avversa l'accusa di essere consociativo. Strano destino. Il successo ha un prezzo per gli uomini come per i concetti: coniato da Lijphart (1968) per rendere conto dell'esistenza di democrazie stabili e relativamente felici, che la scienza politica degli anni '50 (Almond 1956) ignorava e per le quali al tempo stesso prevedeva un tribolato avvenire, il termine consociativismo ha assunto nel nostro paese una connotazione quasi esclusivamente negativa. Consociativa è la spartizione partitica, la corruzione, l'immobilismo, la mancanza di rigore finanziario. In breve, consociativa pare essere stata tutta la Prima Repubblica e consociativi tutti i guai da essa prodotti.

Strano destino davvero, se si pensa che nell'originaria tipologia di Lijphart l'Italia non figurava fra le democrazie consociative e che per molto tempo non fu al concetto di consociativismo che si rivolsero studiosi e commentatori della scena politica italiana. Negli anni '70, perfino quando la grande coalizione fra democrazia cristiana e partito comunista parve a molti ad un passo dalla consacrazione definitiva (ovvero quella governativa), non erano rari fra i politologi perplessità e scetticismo sulla natura e sulla effettiva tenuta dell'accordo.

Questo lavoro ba usufruito dei finanziamenti Cnr per la ricerca «Le politiche pubbliche nello Stato Contemporaneo tra vincoli istituzionali e culturali» diretta dal prof. Giorgio Freddi (contratto 93.018121).

RIVISTA ITALIANA DI SCIENZA POLITICA / a. XXVII, n. 3, dicembre 1997 
Nel 1974 Sartori, ribadendo l'importanza dell'immagine elettorale dei principali partiti rispetto ai comportamenti parlamentari, riproponeva il modello del pluralismo polarizzato nato per spiegare, insieme al caso italiano (e la compagnia era tutt'altro che rassicurante e certo poco «consociativa»), la Germania di Weimar, la Francia della IV Repubblica, la repubblica spagnola fra il 1931 ed il 1936 ed il Cile (Sartori 1974).

Di Palma, nel 1977, distingueva fra segmentazione etnica o linguistica e polarizzazione ideologica per sostenere l'impossibilità in Italia di un assetto consociativo simile a quello attribuito da Lijphart alle piccole democrazie europee (Di Palma 1978).

Pappalardo, nel 1979, dubitava della compattezza e della fedeltà di militanti ed elettori di una e dell'altra parte qualora le leadership partitiche democristiane e comuniste avessero proseguito l'avvicinamento delle rispettive posizioni politiche. Ossia negava l'esistenza dei requisiti necessari all'accordo consociativo (Pappalardo 1979).

Da allora nessun fatto nuovo ha avvicinato l'Italia al modello consociativo, tanto meno il ritorno all'opposizione del partito comunista e i governi craxiani degli anni ' 80 .

A cambiare sono stati l'interpretazione prevalente di fenomeni già prima ampiamente conosciuti, e la stessa tipologia di Lijphart. La testimonianza migliore della nuova «consapevolezza» la offre in un incipit quasi solenne Pizzorno $(1993,285)$ :

Chi, per decenni, avesse osservato i protagonisti che si muovevano sulla scena politica italiana, ascoltato le loro presentazioni di se stessi e le caratterizzazioni che davano gli uni degli altri, non avrebbe potuto non pensare che il destino della prima repubblica italiana era quello di venire lacerata da scontri fra parti irriducibilmente nemiche. Solo ora, al tramonto, ci accorgiamo che tutti i suoi mali provenivano dalla strana, ma, vedremo, spiegabilissima circostanza, che quelle parti, da tempo ormai, anzi, fin quasi dall'inizio, non erano affatto nemiche, bensì amicissime tra di loro, e assai capaci di accordarsi senza troppe remore di sorta.

La tipologia di Lijphart nel frattempo si era trasformata ritornando all'originario dualismo di Almond, scevro però da ogni sfumatura prescrittiva (e vagamente etnocentrica) del primo. L'insistenza dello studioso olandese sulla propensione all'accordo delle élites per spiegare il successo delle democrazie austriaca, belga, olandese e svizzera si era trasformata, nel volume comparato del 1984, in una paziente ricognizione delle diverse caratteristiche istituzionali dei regimi democratici. I due modelli polari proposti, quello consensuale e quello maggiorita- 
rio, erano risposte istituzionali altrettanto efficienti a contesti sociali e culturali diversi (Lijphart 1988, 13):

Il modello di democrazia maggioritaria si rivela particolarmente adatto alle società omogenee, ed in queste funziona meglio, mentre il modello consensuale è più adatto alle società plurali. Per il resto, il rendimento delle democrazie analizzate varia poco.

La democrazia italiana apparteneva a pieno diritto alla classe delle democrazie quasi-consensuali, in compagnia di molti, forse troppi regimi democratici, tutti «rispettabili»; tutti, tranne la IV Repubblica francese, ancora funzionanti, e nessuno in procinto di spegnersi per opera di una giunta militare.

Non meraviglia che una simile conclusione non abbia suscitato entusiasmi ed interesse fra i politologi italiani. Alcuni di loro erano impegnati a sostenere una riforma che rivitalizzasse il meccanismo della responsiveness atrofizzato dalla permanenza quarantennale dello stesso partito al governo: una nuova legge elettorale che rendesse chiare agli elettori le responsabilità politiche delle scelte governative. La democrazia italiana era per costoro sì consensuale, anzi consociativa, ma ormai suo malgrado, perché nel frattempo la società civile si era profondamente evoluta e gli steccati ideologici erano in via di dissoluzione. Una vetusta armatura istituzionale imprigionava una realtà politica e sociale nuova, nuove regole maggioritarie avrebbero permesso di identificare facilmente la paternità politica di decisioni rapide, univoche e alla lunga più efficienti. Scrive di recente Fabbrini $(1994,42-43)$ :

Dopo tutto, per troppo tempo si era pensato che portare a compimento la democrazia italiana significasse associare tutti i principali gruppi o schieramenti politici nella condivisione consociativa del potere esecutivo, piuttosto che cambiare il modello di democrazia. Eppure, l'Italia da tempo è pronta per sperimentare una democrazia competitiva, perché mezzo secolo di stabilità democratica, insieme alla secolarizzazione e modernizzazione della sua società, hanno portato al ridimensionamento delle sue tradizionali divisioni ideologiche (...) e all'affermazione di una cittadinanza democratica, capace di sostenere l'alternanza al governo di schieramenti (politico-programmatici) alternativi.

Così la storia del (termine) consociativismo in Italia, al culmine del suo poco onorevole ma indiscutibile successo, appare paradossale: quando l'Italia sembrava ai più differente dalle piccole democrazie per le quali era stato coniato il termine, il consociativismo era prospettiva tutt'altro che disprezzabile ma poco probabile; quando si iniziò a interpretare il sistema politi- 
co italiano come consociativo, consociativismo divenne sinonimo di tutte le inefficienze e nefandezze del regime.

Le alterne sfortune del consociativismo non sono una bizzarria dei politologi ma sono implicitamente o esplicitamente legate alla spiegazione dell'attività legislativa. La democrazia consociativa nella sua accezione originaria era caratterizzata essenzialmente da una prioritaria cooperazione fra le élite di subculture separate e mutuamente ostili, posta in essere con il deliberato intento di controbilanciare le tendenze disgreganti insite nei sistemi frammentati. Fra le modalità fondamentali di questa cooperazione c'è l'esistenza di grandi coalizioni ministeriali che coinvolgono i leader politici di tutti i segmenti significativi della società, senza distinzione fra maggioranza ed opposizione. Chi avesse esaminato il sistema politico italiano alla luce di questa modalità stricto sensu non avrebbe potuto considerare la democrazia italiana consociativa. Il partito comunista non era mai andato oltre il sostegno parlamentare al governo per un periodo di tempo circoscritto.

D'altra parte, l'importanza del parlamento nel policy making e l'ampiezza delle coalizioni che sostenevano gran parte della legislazione spingevano a quella interpretazione. Il ruolo assegnato al parlamento era insomma cruciale ed è quindi all'attività legislativa del parlamento italiano che rivolgerò le mie attenzioni.

Nel primo paragrafo di questo lavoro accennerò ad alcuni dati sulla produzione legislativa, la maggior parte dei quali ampiamente noti in letteratura. Del fenomeno che descrivono sono state proposte due interpretazioni opposte, l'una che privilegia la lettura polarizzata, e quindi l'assenza di reale consociativismo nella vicenda italiana, l'altra che, al contrario, relega la polarizzazione al ruolo di utile finzione. Cercherò di evidenziare i punti deboli di entrambe le posizioni. Argomenterò a favore dell'efficacia esplicativa di alcuni modelli analitici «individualisti» e razionalisti, nati nel contesto politico statunitense, considerato peraltro distante da quello italiano in tutte le principali tipologie dei regimi democratici ${ }^{1}$. Nel secondo paragrafo illustrerò la logica sottesa a tali modelli ed i loro principali risultati. La tesi contenuta in questa implicita comparazione è che alla contrapposizione

1 Meritevoli eccezioni sono quelle di Cotta (1994b), in cui si sostiene la somiglianza del parlamento italiano al Congresso statunitense, e di Tsebelis (1995), in cui si sostiene la somiglianza del sistema politico statunitense al sistema politico italiano rispetto alla stabilità delle politiche. 
fra modelli di democrazia non corrisponda necessariamente una effettiva contrapposizione dei processi decisionali concreti. Conflitto e cooperazione, frammentazione e coordinamento attraversano diversi sistemi politici. Nel terzo paragrafo proporrò un'interpretazione del processo legislativo del parlamento italiano come l'effetto di un gioco intrecciato fondato sul funzionamento congiunto del mercato elettorale e delle regole parlamentari.

\section{Polarizzazione e consenso, una falsa antinomia}

Fin dal lontano 1963 le ricerche sulla produzione legislativa italiana mostrarono un fenomeno sorprendente: nonostante il clima acceso del dibattito pubblico e benché alle principali forze politiche sembrassero corrispondere irriducibili scelte di civiltà, la maggior parte delle leggi approvate in parlamento venivano votate quasi all'unanimità ${ }^{2}$.

Le coalizioni «parlamentari» contraddicevano i ruoli assunti dai diversi partiti sulla scena elettorale. Il fenomeno era ancora più rilevante se si considera che anche l'iniziativa legislativa del governo, perfino nella forma perentoria del decreto legge, divenne, col tempo, sempre meno la semplice trascrizione dell'indirizzo politico di uno schieramento, e sempre più la mossa di apertura o di chiusura di un gioco complesso di mediazioni e aggiustamenti, che si concludeva spesso con il consenso di quasi tutti i parlamentari coinvolti: consenso all'approvazione della proposta di legge modificata rispetto all'originale o consenso per il suo effettivo accantonamento (Giuliani 1996).

I dati contenuti nelle tabelle 1-4 sono fin troppo noti per meritare altri commenti ${ }^{3}$. Ancora più difficili da spiegare, per chiunque cerchi una qualche corrispondenza fra maggioranze governative o politiche e coalizioni «legislative», sono le cifre riguardanti le iniziative sottoscritte da più di una formazione politica, dove prevalgono coalizioni di proponenti prossime all'unanimità.

Le proposte di legge presentate alla Camera, sottoscritte da esponenti della democrazia cristiana e del partito comunista e approvate definitivamente, sono, in media, sulle tre legislature

2 Si veda Somogyi, Lotti, Predieri e Sartori (1963).

3 Per un'ampia illustrazione e disamina dei dati sulla produzione legislativa vedi De Micheli (1997). 
TAB. 1. Percentuale media di voti favorevoli per un campione di leggi per 7 legislature

\begin{tabular}{lc}
\hline Legislatura & Voti favorevoli \% \\
\hline I & 78,4 \\
II & 83,4 \\
III & 88,3 \\
IV & 76,6 \\
V & 75,3 \\
Media I-V & 81,3 \\
IX & 79,8 \\
X & 88,3 \\
\hline
\end{tabular}

Fonte: I-V legislatura, Di Palma (1978); IX-X legislatura, De Micheli (1994).

TAB. 2. Percentuale di voti contrari o astensioni a seconda del procedimento in un campione di leggi

\begin{tabular}{lcc}
\hline Legislatura & Voti non favorevoli in Aula \% & Voti non favorevoli in Commissione \% \\
\hline I & 25,4 & 10,0 \\
II & 21,8 & 8,4 \\
II & 15,1 & 3,8 \\
IV & 28,4 & 10,7 \\
Media I-IV & 22,6 & 8,2 \\
IX & 29,3 & 11,2 \\
X & 16,0 & 7,2 \\
Media IX-X & 22,6 & 9,2 \\
\hline
\end{tabular}

Fonte: I-IV legislatura, Cazzola (1972); IX-X legislatura, De Micheli (1994).

TAB. 3. Decreti legge emanati, convertiti, emendati per legislatura

\begin{tabular}{lccccc}
\hline Legislatura & Emanati & Convertiti & Convertiti emendati & Conv./tot \% & Emend./conv. \% \\
\hline I & 29 & 28 & 16 & 96,6 & 57,1 \\
II & 62 & 62 & 34 & 100 & 54,8 \\
III & 30 & 28 & 11 & 93,3 & 39,3 \\
IV & 94 & 89 & 49 & 94,7 & 55,1 \\
V & 69 & 65 & 43 & 94,2 & 66,1 \\
VI & 125 & 108 & 74 & 86,4 & 68,5 \\
VII & 167 & 140 & 103 & 83,8 & 73,6 \\
VIII & 284 & 171 & 135 & 60,2 & 78,9 \\
IX & 304 & 136 & 105 & 44,7 & 77,2 \\
X & 470 & 187 & 148 & 39,8 & 79,1 \\
\hline
\end{tabular}

Fonte: I-VII legislatura, Morisi (1992); VIII-X legislatura, dati dell'Archivio elettronico del Senato. 
TAB. 4. Procedura di approvazione alla Camera dei Deputati

\begin{tabular}{lcccccc}
\hline Legislatura & Assemblea & $\begin{array}{c}\text { Assemblea } \\
\text { liberi }\end{array}$ & Commissione & Totale & $\begin{array}{c}\text { Ass./tot. } \\
\%\end{array}$ & $\begin{array}{c}\text { Ass.lib./tot. } \\
\%\end{array}$ \\
\hline VIII & 430 & 13 & 858 & 1.288 & 33,39 & 1,01 \\
IX & 370 & 63 & 847 & 1.217 & 30,40 & 5,18 \\
X & 607 & 140 & 1125 & 1.732 & 35,05 & 8,08 \\
\hline
\end{tabular}

Assemblea liberi: progetti di leggi volontariamente approvati in aula

Totale: Assemblea + Commissione

Fonte: Dati dell'Archivio elettronico del Senato.

considerate, più della metà del numero complessivo di proposte approvate definitivamente e presentate da più di un partito (tab. 5) .

Con una perifrasi poco precisa ma forse efficace, si potrebbero distinguere in letteratura due interpretazioni simmetriche: una lettura «polarizzata» del consociativismo parlamentare ed una «consociativa» della polarizzazione elettorale. La prima è enunciata compiutamente da Di Palma nel suo celebre libro Sopravvivere senza governare (1978). La seconda, con ambizioni interpretative molto più ampie del tema ivi trattato, è stata suggerita recentemente da Pizzorno (1993).

Di Palma non contestava l'esistenza di coalizioni molto estese a sostegno dell'approvazione dei progetti di legge, ma collegava questa apparente anomalia all'irrilevanza di tali provvedimenti. La distanza ideologica che separava gli schieramenti politici in campagna elettorale era la stessa che impediva un accordo sulle questioni «importanti», sulle regole di decisione. Il consenso così vasto attorno a progetti marginali copriva il dissenso profondo sulle grandi riforme e, soprattutto, copriva il dissenso intorno alla legittimità di ciascuna parte dello schieramento politico a decidere, qualora avesse avuto i voti per farlo, senza il consenso dell'altra $(1978,297)$.

La marginalità della maggior parte dei provvedimenti ap-

4 Anche prendendo in considerazione l'universo dei disegni di legge presentati in parlamento, e non solo quelli approvati, il numero dei disegni di legge sottoscritti da DC e PCI resta straordinariamente elevato, nonostante la fase della presentazione, molto più della discussione e votazione, sia quella nella quale più forte dovrebbe essere il bisogno dei gruppi parlamentari di distinzione simbolica. Vedi per un'analisi accurata di tale fenomeno Giuliani (1996, 10-16). 
TAB. 5. Campione delle proposte di legge presentate alla Camera e approvate definitivamente per combinazioni dei proponenti

\begin{tabular}{|c|c|c|c|}
\hline Combinazione proponenti & VIII Leg. & IX Leg. & X Leg. \\
\hline Laici & 12 & 16 & 25 \\
\hline Laici, DC & 3 & 3 & 4 \\
\hline Laici, PCI & 0 & 1 & 0 \\
\hline Laici. PSI & 1 & 3 & 4 \\
\hline Laici, MSI & 0 & 0 & 0 \\
\hline Laici, DC, MSI & 0 & 0 & 1 \\
\hline Laici, DC, PCI & 1 & 0 & 1 \\
\hline Laici, DC, PSI & 7 & 17 & 14 \\
\hline Laici, DC, PCI, PSI & 18 & 16 & 32 \\
\hline Laici, DC, MSI, PSI & 0 & 0 & 4 \\
\hline Laici, DC, MSI, PCI & 0 & 0 & 1 \\
\hline Laici, DC, MSI, PSI, PCI & 11 & 8 & 25 \\
\hline DC & 78 & 85 & 151 \\
\hline DC, PCI & 0 & 1 & 4 \\
\hline DC, PSI & 9 & 3 & 12 \\
\hline DC, MSI & 0 & 1 & 1 \\
\hline DC, PCI, PSI & 7 & 5 & 28 \\
\hline DC, PSI, MSI & 0 & 0 & 1 \\
\hline DC, PCI, MSI & 0 & 0 & 0 \\
\hline DC, PCI, PSI, MSI & 1 & 0 & 4 \\
\hline PCI & 31 & 30 & 106 \\
\hline PCI, PSI & 3 & 4 & 1 \\
\hline PCI, MSI & 0 & 0 & 1 \\
\hline PCI, PSI, MSI & 0 & 0 & 0 \\
\hline PSI & 18 & 34 & 109 \\
\hline PSI, MSI & 0 & 1 & 2 \\
\hline MSI & 9 & 22 & 33 \\
\hline Altri & 8 & 9 & 18 \\
\hline Tot. & 217 & 259 & 582 \\
\hline Proposte di coalizione & 61 & 63 & 140 \\
\hline Proposte DC+PCI & 37 & 29 & 92 \\
\hline Proposte DC+PCI/Tot. & $17,5 \%$ & $11,6 \%$ & $15,8 \%$ \\
\hline Proposte di coalizione/Tot. & $28,1 \%$ & $24,3 \%$ & $24,0 \%$ \\
\hline
\end{tabular}

Fonte: Rielaborazione su dati dell'Archivio elettronico del Senato.

provati dal parlamento è ricavata dall'applicazione di criteri di valore presentati come naturali e non problematici: la platea di beneficiari di ciascuno di tali provvedimenti è ristretta; i provvedimenti non sono per lo più contenuti nei programmi elettorali; gli stessi parlamentari, così solleciti ad approvarli, ne parlano con fastidio ed imbarazzo. Questi criteri di valore, adottati dall'analista, non ci dicono quali siano le motivazioni dei legislatori. Nulla vieta di pensare che le leggi cosiddette sezionali o 
microsezionali corrispondano effettivamente agli obiettivi principali dei loro proponenti. Né, d'altra parte, si può negare che l'effetto complessivo di tali provvedimenti fosse imponente dal punto di vista finanziario o efficace da quello elettorale. L'anello concettuale che lega la polarizzazione del sistema partitico allo stile consensuale delle decisioni parlamentari è la tesi implicita che le decisioni su cui i partiti pervengono ad un accordo siano secondarie anche dal loro punto di vista. E tuttavia le uniche possibili (Di Palma 1978, 244-246).

La tesi di Pizzorno è speculare a quella di Di Palma. Quel che per il primo è inazione dovuta ad un disaccordo di fondo, per il secondo è collusione. La polarizzazione rende il servigio di stabilizzare i mercati partitici, costringe l'attenzione di commentatori (compresi i politologi) ed elettori verso la tenzone combattuta sul palcoscenico elettorale, mentre nelle quinte parlamentari (e non solo in quelle) si consuma lo scambio di favori, la divisione delle spoglie. La prospettiva di Di Palma viene così completamente ribaltata: ciò che conta davvero per i partiti è l'accordo ed i benefici che a ciascuno reca, mentre lo scontro inscenato davanti alla pubblica opinione è solo un'utile finzione.

Quel che differenzia l'interpretazione di Di Palma da quella di Pizzorno è la diversa identità attribuita ai partiti. Per il primo, conta l'identità battagliera delle campagne elettorali; per il secondo, quella collusiva delle decisioni parlamentari (e non solo di quelle). Ciascuna delle identità secondarie nelle rispettive interpretazioni viene considerata funzionale al mantenimento di quella principale.

Quel che accomuna entrambe le interpretazioni è il ruolo assegnato ai partiti. Sono le interazioni fra partiti che spiegano le caratteristiche del law making. Poco importano le regole interne al parlamento5; le caratteristiche stesse del sistema elettorale contano solo per il loro legame con il sistema di partito, mentre è tralasciata l'analisi dei vincoli e delle opportunità che qualsiasi sistema elettorale impone e offre al singolo candidato e/o parlamentare. Se nel caso di Pizzorno l'ambizione di interpretare il funzionamento complessivo del sistema politico italiano della prima repubblica giustifica il silenzio su elementi speci-

5 Nel libro del '77, tradotto in italiano l'anno successivo, Di Palma si sofferma lungamente nella descrizione degli interna corporis del parlamento e sembra attribuire loro una certa importanza. L'interpretazione generale, esplicitamente sartoriana, sembra tuttavia prescinderne completamente. 
fici di questo $^{6}$, nel libro di Di Palma la consapevolezza della loro esistenza è accompagnata da un giudizio di sostanziale irrilevanza.

Per avvalorare la tesi che è il funzionamento del sistema di partito a spiegare l'alluvione di leggi e leggine approvate dal parlamento italiano pressoché all'unanimità, occorrerebbe che sistemi di partito analoghi a quello della prima repubblica italiana avessero generato risultati simili e che sistemi di partito agli antipodi per formato e meccanica fossero all'origine di uno stile decisionale completamente diverso. Questa evidenza empirica non è mai stata prodotta, né è producibile: alle stesse tipologie di parlamento citate successivamente da Di Palma (1987) appartengono paesi con sistemi di partito molto diversi. Il caso statunitense è da questo punto di vista esemplare. Fin dagli anni ' 60 , è stato coniato il termine «universalism» (la cui traduzione letterale è ingannevole) per definire la particolare forma di «consociativismo parlamentare» che caratterizza una parte rilevante dell'attività legislativa interna alle commissioni della $\mathrm{Ca}$ mera dei Rappresentanti; il termine reciprocity definisce invece la mutua deferenza che consente a ciascuna commissione di ottenere l'approvazione dall'aula delle proprie proposte in cambio di un comportamento di analogo rispetto e non interferenza per proposte provenienti da altre commissioni. Universalism e Reciprocity ${ }^{7}$ sono i concetti chiave del textbook sul Congresso fino alla fine degli anni $70^{8}$. Ovvero, sono le caratteristiche più salienti dello stile decisionale adottato da un parlamento di una democrazia definita negli stessi anni da Lijphart come depoliticizzata, la cui dinamica partitico-elettorale sembrava rispettare, nel complesso, la scarna rappresentazione downsiana di una incoercibile tendenza delle forze politiche a collocarsi, se non addirittura a sovrapporsi e confondersi, al centro del continuum

6 Pizzorno chiama a sostegno della sua ipotesi, oltre alle ampie maggioranze formatesi nelle commissioni parlamentari, anche elementi tipici di quello che altri hanno chiamato governo di partito spartitorio: questi ultimi elementi appaiono in realtà meno probanti dei primi. Nella maggior parte degli enti pubblici e delle banche la spartizione delle poltrone avveniva fra i partiti che sostenevano la compagine governativa. Le eccezioni riguardavano enti particolari come l'INPS (che per statuto accoglieva ai suoi vertici rappresentanti sindacali) o la Rai, il cui controllo era istituzionalmente delegato al parlamento.

7 Vedi Fiorina (1981).

8 Per una ricostruzione dell'evoluzione del congresso dagli anni ' 70 alla fine degli anni '80 vedi Shepsle (1989). 
ideologico destra-sinistra 9 . Si può obiettare che il sistema politico statunitense è troppo diverso da quello italiano, ed altri sono gli elementi che concorrono a determinare esiti per certi versi analoghi: il rapporto fra presidenza e Congresso, la debolezza organizzativa dei partiti, l'assetto federale, etc. La replica, tanto ovvia quanto sensata, se condotta fino alle sue estreme e logiche conseguenze non è tanto una difesa delle spiegazioni «partitiche» dell'attività legislativa del parlamento italiano quanto piuttosto il loro de profundis. Se infatti siamo costretti a chiamare in causa una molteplicità di fattori per spiegare il caso statunitense, non si comprende perché altrettanto non si debba fare per quello italiano. Resta inoltre l'enigma della relativa somiglianza dei processi decisionali nei due casi, o per lo meno della adattabilità di alcuni dei resoconti pensati per quel contesto al contesto italiano. Questo dato è ancora più difficile da spiegare per quanti interpretano il caso italiano come l'eccezionale, irripetibile prodotto di un processo di formazione dello stato-nazione ritardato e forse mai concluso. La nostra tesi generale va in tutt'altra direzione: non sono le singole caratteristiche del sistema politico-istituzionale che possono spiegare le caratteristiche dei processi decisionali in parlamento. Non è la legge elettorale, non è il sistema di partito, non sono le regole interne alle camere né è il rapporto fra governo e parlamento. È il modo in cui queste ed altre caratteristiche si compongono insieme. Regole molto diverse fra loro possono formare costellazioni istituzionali dagli esiti simili. E a costellazioni istituzionali fra loro molto simili, ma diverse per una qualche regola, sono associati con ogni probabilità esiti anche molto diversi fra loro. L'algebra delle istituzioni non si esaurisce in una semplice sommatoria di caratteristiche il cui segno e la cui importanza è data a priori. Una descrizione sommaria degli elementi politico-istituzionali di una democrazia rischia insomma di essere fuorviante quando sulla base di questa descrizione si tentano spiegazioni, o peggio, previsioni del tipo di law-making in parlamento. D'altra parte,

9 Provate a confrontare questa frase tratta da Brady $(1988,1)$ con quella seguente di Di Palma (1978, 134): «Il processo decisionale nel Congresso talvolta assomiglia ad un tritacarne che riduce le grandi questioni pubbliche ad una serie di singoli, scollegati e spesso contraddittori pezzi di politica»; «Quando un'istituzione è incapace di formare solidi accordi intorno a decisioni difficili, essa non desiste dal decidere, ma adatta piuttosto la sua produzione ed i suoi procedimenti alle circostanze: disaggrega la maggior parte delle sue decisioni, postpone quelle più difficili, reagisce ed adatta, invece di iniziare e rinnovare...». 
non è chiaro quando una simile descrizione diventa esaustiva: la ricerca dei dettagli potrebbe rivelarsi ben presto un compito interminabile e le congetture avanzate sulla base di descrizioni incompiute potrebbero apparire sempre provvisorie.

Per poter uscire da questo dilemma si deve abbandonare una ricerca delle caratteristiche istituzionali ancora troppo debitrice nei confronti della tradizione giuridica: non è infatti attraverso una ricognizione, non importa quanto precisa, delle regole di un assetto istituzionale che ne possiamo comprendere il funzionamento. Non solo perché non tutte le regole sono egualmente «in uso» ma anche perché senza il riferimento all'attore politico i cui comportamenti le regole vincolano, non possiamo comprendere fino in fondo il funzionamento congiunto delle stesse regole. E l'attore politico ultimo convenga che sia l'individuo. In altre parole, occorre, a mio avviso, procedere dai singoli individui intesi come attori politici unitari ed irriducibili ad altro ed osservare rispetto alle loro azioni quali sono i concreti sistemi di vincoli ed opportunità. Solo in un secondo tempo potremo ricostruire l'interazione fra organismi sovraindividuali, tenendo ben presente che non si tratta dell'interazione fra attori collettivi dotati di intenzionalità ma semmai dell'interazione fra attori individuali in grado di prendere decisioni che vincolano $\mathrm{i}$ comportamenti dei membri del collettivo a cui essi stessi appartengono. Questa capacità (di vincolo) non è a sua volta data una volta per tutte ma è il risultato, sempre contingente e provvisorio, del funzionamento congiunto del complesso di regole, interne ed esterne a quel collettivo. Un punto di vista individualistico consente di sfuggire alla reificazione delle definizioni dei soggetti politici: il peso di una passata tradizione di ricerca o di una brillante ed efficace trattazione di tematiche contigue a quelle oggetto di studio, possono infatti ingenerare l'abitudine a pensare nei termini di determinati attori, la cui supposta presenza e le cui presunte caratteristiche si sono rivelate in alcuni casi illuminanti ed efficaci nel corso della spiegazione. Sennonché l'una e le altre, ossia quella presenza e quelle caratteristiche, sono imprescindibilmente legate a quei casi e a quella spiegazione. Così se l'attore partito si è rivelato fondamentale nella spiegazione delle caratteristiche assunte dalla transizione alla democrazia nel caso italiano, questo non significa a priori che ricopra il medesimo ruolo nella spiegazione dell'attività legislativa. Seguendo un approccio individualistico potrò sempre scoprire che l'ipotesi di un ruolo fondamentale delle decisioni pre- 
se (o dei veti posti) dalla leadership di partito nel law-making è corroborata dall'evidenza empirica. Viceversa se il mio punto di partenza è il partito (o la commissione, o i gruppi d'interesse ecc.), rischio di offuscare e cancellare, già in fase di impostazione della ricerca, il ruolo dei singoli e degli altri collettivi di riferimento non considerati.

Si può studiare il funzionamento di un'istituzione dal punto di vista dell'individuo attraverso una ricerca empirica di stampo comportamentista: si indaga attraverso questionari e interviste, sulle intenzioni dei singoli, e si cerca di ricostruire, sulla base delle risposte e delle informazioni di contesto raccolte, quale sia la rete di relazioni fra gli attori, le loro competenze, le caratteristiche dell'effettivo processo decisionale. Oppure, si può adottare un approccio deduttivo, attribuendo agli attori determinati scopi e costruendo modelli esplicativi che, sulla base di quegli scopi, rendono conto degli eventi. In quest'ultimo caso non si rinuncia all'indagine empirica: poiché la ricostruzione delle circostanze concrete in cui si muovono gli attori è fondamentale nella formulazione del modello, anche in questo caso si potrà far buon uso di interviste, di resoconti giornalistici, di dichiarazioni ufficiali ed ufficiose. La differenza fondamentale fra i due approcci sta nel diverso modo di intendere gli scopi degli attori: nel primo caso si intendono per scopi gli atteggiamenti, e la scelta di alcuni a scapito di altri si fonda sulla verifica empirica. Una volta ascoltati dalla viva voce delle persone, devono essere inclusi nel modello esplicativo. Nell'approccio deduttivo-formale, al contrario, non si ha una pretesa assoluta di realismo nella scelta degli scopi da attribuire agli attori. Il realismo degli scopi, che in questo caso sono preferenze, è un prodotto dell'efficacia del modello esplicativo.

All'apparenza il metodo deduttivo sembrerebbe assai più debole. Esistono tuttavia ottime ragioni per sostenere il contrario: 1) affidarsi completamente alle risposte degli intervistati trascura l'eventualità che quelle stesse risposte siano comportamenti strategicamente orientati; 2) quand'anche le risposte fossero «sincere», esse rifletterebbero contemporaneamente sia la struttura motivazionale dell'individuo che quella cognitiva, ovvero sia le preferenze che le credenze, e non esiste nessuno stratagemma della ricerca empirica in grado di separare le une dalle altre. In altre parole, il ricercatore dispone non solo dell'insieme dei desideri degli attori, ma anche di un insieme di giudizi impliciti sulla loro realizzabilità. Ogni qualvolta si modifica 
(esogenamente o endogenamente) il sistema di vincoli ed opportunità, rischiamo di essere costretti a ridescrivere gli attori stessi poiché è probabile che anche i loro stessi atteggiamenti si siano modificati. $\dot{E}$ chiaro che in tal modo si possono solo registrare i mutamenti, senza mai renderne conto teoricamente. Se invece disponiamo di una descrizione sufficientemente stabile delle preferenze degli attori (delle loro identità) possiamo tentare di spiegare i diversi comportamenti da loro esibiti, ed il diverso funzionamento di un sistema di interazione, come un adattamento al mutato sistema di vincoli ed opportunità, alla mutata distribuzione di risorse ${ }^{10}$.

Un buon esempio di scopo assegnato a priori per convenienze analitiche, prima ancora che per realismo, è lo scopo della rielezione per i parlamentari. I parlamentari non perseguono solo - né, in alcuni casi, principalmente - lo scopo della rielezione. Di certo non molti sarebbero disposti a confessarlo ad un intervistatore, anche quando questi fosse circospetto e abile nel porre le domande. Nei modelli razionali-deduttivi impiegati per lo studio del Congresso, tuttavia, lo scopo della rielezione è un ingrediente fondamentale, e tale sarà implicitamente anche per me nelle prossime pagine. La rielezione è uno scopo plausibile, e sufficientemente diffuso. Non occorre aderire ad una visione cinica della politica per immaginare che i politici si preoccupino della loro sopravvivenza nel circuito decisionale parlamentare: se anche hanno a cuore $\mathrm{i}$ destini della collettività di cui sono parte e non (o non solo) il prestigio e gli emolumenti che la carica assicura, se ritengono che il loro contributo sia importante, cercheranno innanzitutto di essere rieletti. Si può ipotizzare che

10 Si può anche supporre che il legame fra determinate risorse e gli scopi che l'attore intende perseguire con le stesse sia a tal punto forte, dal punto di vista dell'attore, da impedirne una sostituzione. In altre parole si può immaginare che l'identità dell'attore non sia definita solo dagli scopi ma pure da determinati modi in cui tali scopi sono conseguiti. Questo comporta però una scomparsa dell'attore medesimo, che rinuncia, per salvare se stesso, a realizzare i propri scopi e quindi ad esistere nella sua originaria definizione. Un politico abituato ad utilizzare le sottigliezze e le oscurità del discorso ideologico per ottenere il necessario consenso alla sua azione politica, può rifiutarsi, in un contesto di accresciuta esposizione dei politici ai mezzi di comunicazione di massa e di ridotta militanza, di prestare attenzione alla propria immagine e di mutare il suo stile comunicativo. Se questo comportamento «conservatore» non è a sua volta un'abile forma di differenziazione dell'offerta politica e quindi di cattura alternativa del consenso, è probabile che alla lunga conduca alla sparizione di quel politico, sostituito da qualcuno in grado di operare efficacemente nelle mutate condizioni. Tale fenomeno di sostituzione di uomini può, dal punto di vista analitico, essere descritto come una forma di adattamento del medesimo attore. 
questa preoccupazione guidi il loro comportamento in misura variabile, a seconda del consenso già raccolto e della sua stabilità, nonché della anzianità parlamentare: la preferenza per la rielezione è infatti una preferenza temporale per il futuro.

I modelli costruiti sullo scopo della rielezione fissano insomma i limiti della loro applicabilità, come qualsiasi altro modello volutamente parziale.

\section{Universalismo e politiche distributive nel Congresso americano}

I seguaci dell'approccio razionale allo studio della politica che rivolsero per primi la loro attenzione alle vicende del Congresso si imbatterono in due fenomeni che dal punto di vista della teoria erano paradossali. Nel Congresso si prendevano decisioni, e spesso a larghissima maggioranza. Gli stessi fenomeni suscitavano molta meno sorpresa fra gli scienziati politici tradizionali: il primo sembrava tanto ovvio da non richiedere alcuna spiegazione: cos'altro avrebbe dovuto succedere in un'assemblea eletta per legiferare? Il secondo, ovvero che il processo decisionale mostrava un inatteso livello di consenso fra i deputati, poteva essere facilmente spiegato con la scarsa conflittualità ideologica, la prossimità del programma democratico a quello repubblicano. Gli allievi di Riker dovevano invece conciliare $\mathrm{i}$ due fenomeni con due risultati della teoria razionale del voto molto robusti, che non concedevano plausibili eccezioni: 1) il teorema di impossibilità di Black-Arrow ${ }^{11}$; e 2) il teorema della coalizione minima vincente (size principle) (Riker 1962).

Per il primo teorema, non appena il numero di votanti e di alternative è pari o superiore a tre, è logicamente possibile - e all'aumentare delle alternative e dei votanti sempre più probabile incorrere in maggioranze cicliche: nessuna alternativa è destinata ad ottenere una maggioranza stabile di consensi, a meno che tutti i votanti non condividano un medesimo criterio di valutazione; ovvero a meno che lo spazio politico sia unidimensionale.

Per il secondo teorema una qualsiasi alternativa, quand'anche risulti stabilmente sostenuta da una maggioranza, dovrebbe essere sostenuta da una maggioranza minima, appena sufficien-

11 La letteratura sull'argomento è sterminata. Oltre ad Arrow $(1951,1963)$ e Black (1958), occorre segnalare ulteriori generalizzazioni del teorema originario, chiamati anche teoremi del caos, ad opera di Plott (1967), McKelvey (1976) e Schofield (1978). 
te. Il primo paradosso è logicamente più radicale del secondo: se nel secondo a contraddire la teoria è la non prevista dislocazione dell'equilibrio che pure trova riscontro nella realtà empirica, nel primo è la stessa esistenza di un equilibrio a creare sconcerto.

La distanza della realtà empirica dalle previsioni della teoria spingeva a chiedersi che cosa in quella realtà, ossia che cosa nell'attività legislativa del Congresso, permetteva ciò che la teoria escludeva senza incertezze. $\mathrm{O}$, detto in altri termini, quali erano le caratteristiche implicite del teorema di impossibilità e del teorema delle coalizioni minime vincenti che conducevano a risultati irrealistici. Tullock ${ }^{12}$, attratto dalla possibilità di analizzare i comportamenti di voto come fossero transazioni in un mercato, sosteneva che le larghe maggioranze erano il frutto di uno scambio di voti generalizzato (logrolling), in cui ciascuno sacrificava le proprie prime preferenze su questioni per lui di poco conto in cambio di un appoggio su questioni per lui di primaria importanza. La soluzione proposta da Tullock non solo non risolveva il paradosso delle maggioranze cicliche - era infatti presupposto dello stesso logrolling l'esistenza di uno spazio politico (di valutazione) multidimensionale - ma importava nella sfera politica i problemi connessi alla creazione della fiducia e all'esistenza di comportamenti opportunistici, già emersi dallo studio delle transazioni nel mercato.

Nel corso degli anni '80 videro la luce una serie di articoli di Shepsle, Weingast, Fiorina e, da ultimo, Krehbiel ${ }^{13}$ che rendevano per la prima volta conto dei comportamenti legislativi nella Camera dei Rappresentanti da un punto di vista razionale. L'idea generale era di incorporare negli scarni modelli fino ad allora elaborati una serie di caratteristiche istituzionali che possedessero come requisito la proprietà di indurre l'equilibrio, non garantito invece dalle rappresentazioni più astratte della situazione di scelta, senza per altro determinarne univocamente il contenuto $^{14}$. La strategia di ricerca consisteva innanzitutto nel definire con precisione quali caratteristiche avrebbe dovuto avere una determinata regola od una determinata circostanza per

12 Si veda Tullock (1984 e 1981).

13 Weingast (1979), Shepsle (1979a e 1979b), Fiorina (1981), Shepsle e Weingast (1981a e 1981b), Krehbiel (1987 e 1988).

14 L'equilibrio reso possibile dalla struttura è infatti determinato dalla distribuzione delle preferenze. 
costringere il modello che ne avesse incorporata l'esistenza a produrre una soluzione simile agli effettivi accadimenti (e quindi, prima di ogni altro, l'esistenza di un equilibrio decisionale). Il secondo passo consisteva nel ricercare nella realtà del Congresso quella regola o quella circostanza. Le modificazioni introdotte trasformavano lo spazio multidimensionale delle politiche in una situazione di scelta unidimensionale; limitavano la convenienza di comportamenti opportunistici; allungavano gli orizzonti temporali degli attori trasformando il valore stesso delle poste in gioco; ricostruivano la perduta ratio di certe azioni attraverso la moltiplicazione e l'intreccio delle arene considerate nei modelli, in accordo con la complessità dei contesti in cui tali azioni effettivamente avevano luogo ${ }^{15}$. Questa strategia di ricerca permetteva di valutare il peso di ciascuna regola e circostanza nel rendere possibile l'equilibrio (e quindi la decisione). Inoltre, data una certa distribuzione di preferenze, essa consentiva di determinare dove fosse tale equilibrio, e quindi quale forma ed esito prendesse il processo decisionale.

Alla fine degli anni ' 80 , il bagaglio di analisi accumulato ${ }^{16}$ permetteva di rendere conto di fenomeni altrimenti inspiegabili all'interno dell'approccio razionale.

Data una certa distribuzione di preferenze di policy, la cui determinazione, in virtù del «nesso elettorale», era fatta dipendere dalla natura delle constituencies rappresentate, si poteva sostenere coerentemente con gli assunti dell'approccio che:

1) la mutevole frequenza di comportamenti di voto strategici (sofisticati) anziché sinceri dipendeva dalla visibilità dei singoli parlamentari di fronte alle rispettive constituencies e dalla numerosità del consesso in cui tali comportamenti avevano luogo: meno controllo il singolo aveva sui risultati del processo decisionale e più visibili erano i suoi atti all'esterno, più probabile era un comportamento sincero, preoccupato della fedeltà ai propositi enunciati e indifferente alle conseguenze sul contenuto finale delle decisioni (Denzan, Riker e Shepsle, 1985);

2) la possibilità di un equilibrio decisionale era indotta dall'assetto organizzativo e dalla divisione del lavoro interni alla Camera dei Rappresentanti e/o dalle procedure di votazione: le prime riconducevano lo spazio multidimensionale delle varie

15 A tal proposito vedi Tsebelis (1990).

16 Per una sintesi vedi Strom (1990). 
policies ad uno spazio unidimensionale delle decisioni, le seconde condizionavano l'agenda setting impedendo la ripresentazione di proposte già sconfitte nel corso delle precedenti votazioni;

3) la frequenza di maggioranze prossime all'unanimità dipendeva da un processo di contrattazione in cui giocavano un ruolo fondamentale la lunghezza degli orizzonti temporali dei decisori, l'incertezza sulla composizione delle coalizioni vincenti ed il costo elettorale di un'eventuale esclusione da queste ultime o, al contrario, i vantaggi della partecipazione.

L'universalismo del processo decisionale e il carattere fortemente distributivo delle policies scaturivano dalla combinazione di regole e circostanze concrete che operavano in modo peculiare sulla propensione a comportamenti strategici, sulla dimensionalità dello spazio di policy e sulla limitazione delle alternative in agenda, sull'incertezza rispetto alle coalizioni sperimentata dai singoli legislatori e sui loro orizzonti temporali.

Innanzi tutto il sistema elettorale uninominale legava le probabilità di rielezione dei singoli deputati agli interessi prevalenti o maggiormente attivi nel collegio elettorale. Lo stesso sistema elettorale impediva che nei medesimi luoghi decisionali sedessero potenziali competitori (per ogni collegio un solo rappresentante).

In secondo luogo, il sistema delle commissioni, ciascuna con una propria giurisdizione, favoriva la specializzazione nella difesa degli interessi e quindi, in una certa misura, una relativa unidimensionalità dello spazio di policy. L'autoselezione dei parlamentari (Shepsle 1978), indotta dal calcolo elettorale, rendeva particolarmente remunerativo per tutti i componenti della commissione il passaggio del maggior numero possibile di disegni di legge, ottenuto grazie al logrolling interno alla commissione ${ }^{17}$. A sua volta la necessità da parte di ciascuna commissione di ottenere l'approvazione da parte dell'aula spingeva verso la reciprocità, ovvero verso un logrolling esterno, fra commissioni.

In terzo luogo le regole di votazione dei disegni di legge in aula assegnavano un formidabile potere di agenda alle commissioni rispetto all'aula. La capacità da parte di ciascuna commissione di far passare le proprie proposte, e soprattutto di bloccare l'approvazione di quelle fortemente sgradite, favoriva la compatezza interna delle commissioni stesse.

17 L'idea implicita è che lo stesso grado di interessamento per una certa area di policy renda per il singolo deputato meno onerosi elettoralmente $\mathrm{i}$ provvedimenti approvati in favore di constituencies diverse dalla propria. 
Infine il professionismo politico e la stabilità politico-elettorale, allungando l'orizzonte temporale dei parlamentari, rendevano maggiormente remunerativa la collaborazione. D'altra parte, il pericolo di non essere rieletti accresceva l'attivismo dei parlamentari nel promuovere gli interessi della propria constituency e quindi la disponibilità al logrolling.

\section{Una rilettura razional-deduttiva del caso italiano}

Per il lettore italiano quel che colpisce dei modelli razionali è lo scarso ruolo dei partiti. Gli stessi autori citati sono consapevoli del costo di questa assenza in termini di realismo descrittivo; si dimostrano tuttavia ben disposti a pagarlo appellandosi alla vulgata sul sistema politico americano secondo la quale i partiti americani sono deboli, divisi al loro interno e privi di qualsiasi possibilità di condizionare attraverso minacce credibili l'attività legislativa dei singoli congressmen (Mayhew 1974; Weingast e Marshall 1988).

Questa difesa ha tuttavia alcuni limiti: 1) rischia di confinare l'impostazione razionale della ricerca a sistemi politici in cui i partiti hanno le stesse caratteristiche di quelli statunitensi; 2) non rende conto di aree di policy (nel caso statunitense potremmo dire sicuramente «commissioni») in cui il partito o, per lo meno, l'appartenenza ideologica conta; 3) non rende conto di periodi storici in cui negli stessi Stati Uniti i partiti hanno contato $^{18}$.

Il primo punto è quello che mi sta maggiormente a cuore perché il law-making italiano, letto con le lenti dei razionalisti americani, sembra essere un vero e proprio enigma. Per anni la scienza della politica e la pubblicistica italiana hanno insistito ossessivamente sullo strapotere dei partiti, sul loro ruolo abnorme all'interno delle istituzioni e della società civile. E tuttavia logrolling, pork barrel, universalism, sembrano termini nati per definire gran parte dell'attività legislativa del parlamento italiano. Come è possibile?

Dal punto di vista delle teorie razionali, qualsiasi assetto organizzativo in grado di ridurre la multidimensionalità dello spazio di policy e di garantire la contrattazione può spiegare l'equi-

18 Vedi Brady (1988); Shepsle (1989); Cox e McCubbins (1993). 
librio decisionale in un'assemblea rappresentativa. Gli stessi studiosi del Congresso hanno suggerito che in contesti istituzionali molto diversi da quello statunitense, come quello britannico, o nel passato della storia parlamentare americana, i partiti abbiano svolto un ruolo simile. Perché i partiti possano avere un ruolo nell'equilibrio decisionale dobbiamo immaginare che ciascun partito in parlamento, al pari di ciascuna commissione, rappresenti una dimensione differente. Il logrolling avverrebbe pertanto fra e dentro i gruppi parlamentari piuttosto che fra e dentro le commissioni. Oppure possiamo immaginare che la multidimensionalità potenziale dello spazio di policy sia ricondotta all'unidimensionalità della battaglia ideologica-elettorale: in tal caso non esistono spazi di contrattazione ma solo vincenti e perdenti ed un equilibrio prossimo al votante mediano dell'aula.

Nel caso italiano in molti settori di policy è dubbio che siano stati i partiti i protagonisti del consenso legislativo. Il contenuto delle «leggine» non era ovviamente incluso nei programmi di partito. L'accordo non era quindi fra le identità palesi dei partiti. Seguendo Pizzorno, si può ipotizzare che avvenisse fra le loro identità occulte ${ }^{19}$. Perché questa possibilità, almeno nello studio dell'attività parlamentare, sia empiricamente fondata, occorrerebbe che $\mathrm{i}$ responsabili di settore o le figure di maggior rilievo all'interno dei partiti siano stati anche i policy makers più attivi; oppure, occorre che le proposte presentate in commissione da parlamentari di seconda fila siano state in precedenza votate (o decise) all'interno del gruppo parlamentare (o di qualche altro organo partitico); o ancora che la composizione delle commissioni non trovi nessuna spiegazione migliore di quella inscritta nelle imperscrutabili volontà dei leader o degli organi dirigenti partitici ${ }^{20}$. Non disponiamo di alcuna evidenza empirica a sostegno di queste ipotesi. Al contrario, sappiamo da alcune ricerche che esistono aree di policy, come per esempio le politiche pensionistiche, in cui i dipartimenti programmatici dei partiti ed i dirigenti ad essi preposti hanno contato assai poco nel processo decisionale (Regonini 1994 e 1996).

Al termine della X legislatura, solo il $20 \%$ dei disegni di legge parlamentare presentati alla Camera da democristiani e

19 L'uso del concetto di identità palese ed identità occulta richiama da vicino la distinzione sartoriana fra politica visibile e politica invisibile (Sartori 1974). Con un taglio assai diverso e al quale sono molto debitore vedi anche Tsebelis (1990).

20 Vedi Krehbiel (1993). 
convertiti in legge, ed una percentuale analoga di progetti presentati dai comunisti e convertiti in legge, hanno avuto come primo proponente un responsabile degli uffici programmatici del partito o un membro del comitato direttivo del gruppo, presidente e vicepresidente inclusi. I deputati democristiani che ricoprivano cariche al vertice degli organi di partito o nel gruppo parlamentare erano pari al $23 \%$ del totale dei deputati. I deputati comunisti nelle medesime condizioni erano il $28 \%$. D'altra parte, durante la stessa legislatura, il 50\% dei disegni di legge di iniziativa parlamentare presentati alla Camera e convertiti in legge avevano fra $\mathrm{i}$ loro proponenti membri della commissione di assegnazione. Dato che le commissioni sono 13, ed in ciascuna vi è l' $8 \%$ del totale dei deputati, non sembra azzardato concludere che i membri di una commissione in media avevano maggiore influenza sull'iniziativa legislativa assegnata alla propria commissione di quanta non ne avessero i dirigenti del partito e del gruppo parlamentare sull'iniziativa legislativa scaturita all'interno del proprio partito e gruppo parlamentare.

Non possiamo certo sostenere che tutte le proposte di legge eludessero il vaglio dei gruppi parlamentari o di altri organi decisionali partitici: tuttavia il tenore generale della produzione legislativa e la lettura dei verbali di commissione spingono a ritenere che sia stata più spesso quest'ultima, se non addirittura le sue articolazioni interne informali (i comitati ristretti), il luogo di reale contrattazione. Riguardo infine alla composizione (reale) delle commissioni, non esiste a tutt'oggi nessuna ricerca sistematica sui criteri di assegnazione. Il sistema proporzionale con voto di preferenza (al contrario di un sistema uninominale maggioritario) creava constituencies personali dai confini incerti e maldefiniti, ed uno studio sull'eventuale legame fra la natura della constituency del deputato e la commissione di appartenenza presenta non poche difficoltà. Almeno per due commissioni, Agricoltura e Trasporti, la cui giurisdizione riguarda politiche strettamente legate al territorio, questo legame risulta essere stato molto forte (Zucchini 1995).

Quanto più le questioni trattate si allontanano dalle conoscenze ed interessi condivisi dalla maggioranza dei membri parlamentari di un determinato partito, tanto più i membri della commissione diventano i rappresentanti della medesima e degli interessi in essa difesi presso il partito ed il gruppo parlamentare, piuttosto che i rappresentanti del partito e del gruppo parlamentare presso la commissione. 
La rilevanza attribuita ai partiti, più che discendere dall'evidenza empirica, deriva dall'appiattimento dell'arena parlamentare sull'arena elettorale. Se si ritiene che ciò che realmente conta è il mancato accordo sulle grandi questioni, oggetto di scontro nelle campagne elettorali, piuttosto che l'accordo diffuso su argomenti limitati e non controversi, stretto, al riparo da occhi indiscreti, nelle aule parlamentari, allora è abbastanza naturale che i protagonisti del mancato accordo appaiano indirettamente anche la causa degli accordi sulle leggine. Il che è impossibile se si considerano le identità palesi - leggi «elettorali» - dei partiti e privo di sostegno empirico, se si considerano i partiti in parlamento. Nel primo caso perché quelle «microdecisioni» non sono parte della definizione che $\mathrm{i}$ leader di partito danno del partito stesso (né come programma per il futuro, né come rivendicazione dell'opera svolta). Nel secondo perché non sembrano essere il gruppo parlamentare o gli organi dirigenti di partito il luogo principale ove vengono processate le proposte «sezionali» e «microsezionali» sulle quali si raggiungerà un accordo in commissione. La spiegazione «partitica» delle cosidette leggine sembra quasi basarsi, nel caso di Di Palma, su una metafora «idraulica»: il vuoto decisionale per le grandi questioni è necessariamente riempito da decisioni minime e non conflittuali; non interessa sapere quale sia l'effetto complessivo di tali decisioni, quali siano i protagonisti, in che misura il loro successo influenzi la capacità di decidere sulle grandi questioni.

Nonostante questi limiti le spiegazioni di Pizzorno e di Di Palma, opposte e speculari ma accomunate dall'insistenza ad attribuire ai partiti una parte cruciale, colgono, senza svilupparla, la stretta connessione fra le cosiddette grandi questioni, perennemente eluse, e i cosiddetti microprovvedimenti approvati da quasi tutti.

Le loro rispettive posizioni possono essere rilette e ricomposte in un quadro unitario e coerente con l'evidenza empirica attraverso un modello di gioco intrecciato ${ }^{21}$.

Il gioco delle grandi politiche cui accenna Di Palma ha la struttura di un classico dilemma del prigioniero ${ }^{22}$ ed è condotto

21 Il riferimento d'obbligo è in questo caso a Tsebelis (1990) e Scharpf (1990).

22 Lo stesso Di Palma accenna, in tempi in cui la teoria dei giochi era molto meno diffusa in scienza della politica, al dilemma del prigioniero come forma di rappresentazione del clima di sfiducia fra élite $(1978,185)$. 
dai leader partitici. Le mosse a disposizione delle parti sono la Cooperazione, che consiste nel caso dell'attività parlamentare nell'accettazione delle richieste dell'avversario (per esempio nel non frapporre ostacoli procedurali all'approvazione di un disegno di legge fortemente voluto dalla parte avversa), e la Defezione, ovvero un sistematico boicottaggio delle richieste altrui e il tentativo di imposizione delle proprie. Un contesto fortemente polarizzato è allora un contesto in cui l'assenza di decisioni è preferibile alla vittoria dell'altra parte o alla propria sconfitta $(\mathrm{DD}>\mathrm{CD})$, e la propria vittoria è preferibile ad una soluzione contrattata (DC > CC); in altri termini l'ordine di preferenza delle mosse è $\mathrm{DC}>\mathrm{CC}>\mathrm{DD}>\mathrm{CD}$ e la soluzione finale del gioco (l'esito collettivo) è DD, ossia lo stallo decisionale.

D

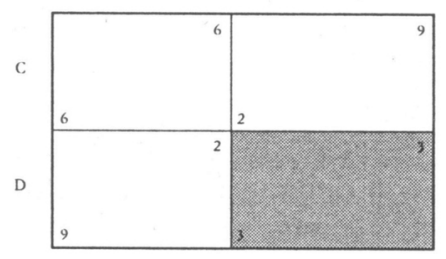

FIG. 1a. Gioco delle grandi politiche23.

Nonostante la soluzione contrattata sia preferibile per entrambi, la reciproca sfiducia conduce allo stallo decisionale (DD). Senonché perché uno stallo decisionale sia preferibile dalle parti alla «vittoria» di una delle due (C-D o D-C) occorre che a tale stallo non corrisponda un pericolo di tenuta del sistema democratico o l'ingresso di un terzo attore partitico in grado di mettere in forse il predominio di una delle due parti. Occorre insomma che allo stallo decisionale corrisponda il mantenimento e consolidamento dei consensi di ciascuna delle parti. Questo è reso possibile dal gioco delle leggine ${ }^{24}$ che ha una struttu-

23 I numeri hanno un'utilità euristica. Quel che conta non è il loro valore assoluto ma i rapporti di diseguaglianza. Per una disamina più accurata e formalizzata dei giochi vedi l'appendice.

24 La distinzione fra gioco delle leggine e gioco delle grandi politiche richiama la distinzione operata da Cotta (1996) fra metapolitiche e micropolitiche. Nell'impostazione di Cotta tuttavia è soprattutto il contenuto delle questioni sulle quali gli attori politici sono chiamati a decidere a definire il tipo di politiche, mentre nella nostra impostazione contano maggiormente il tipo di attori coinvolti e gli ambienti decisionali. 
ra intrinsecamente cooperativa ed è giocato dai singoli parlamentari.

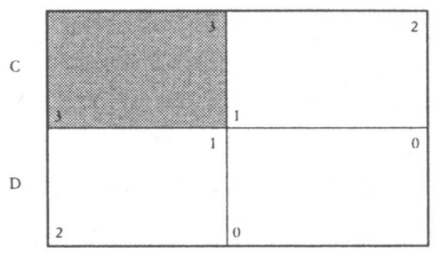

Soluzione del gioco

FIG. 1b. Gioco delle leggine.

Nel gioco delle leggine i singoli parlamentari perseguono la propria soppravvivenza nel circuito decisionale parlamentare e nello stesso tempo producono una quota di consenso che si riversa sul partito in quanto tale, e che costituisce un patrimonio indipendente dalla capacità dei partiti di raggiungere una qualche decisione nel gioco delle grandi politiche. D'altra parte è altrettanto plausibile (ed anche più consueto) supporre che la capacità di raggiungere una qualche decisione nel gioco delle grandi politiche da parte dei leader di partito produca una quota di consenso per i singoli parlamentari che prescinde dalla loro capacità di stipulare accordi in parlamento. Per il suo carattere indifferenziato tuttavia tale quota, non favorendo all'interno del medesimo partito nessun parlamentare contro l'altro, (e al limite neppure un parlamentare in carica rispetto ad un candidato del medesimo partito nella stessa circoscrizione) è probabile non assuma un valore molto alto. Per semplicità espositiva non considereremo queste plausibili diversità di impatto dei risultati di un gioco sui pay-offs dell'altro. L'assenza di decisioni in un'arena non produce ovviamente alcun valore che allevii i costi dello stallo decisionale nell'altra ${ }^{25}$.

Se alla defezione reciproca nel gioco delle «grandi politiche» corrispondono almeno in parte i pay-offs della cooperazione nel gioco delle leggine (vedi fig. 1a e fig. 1b) la domanda a questo punto è: quali sono gli insiemi di regole e circostanze che rendono possibile tale cooperazione? pendice.

25 Per una più sistematica e formalizzata trattazione di entrambi i punti vedi l'Ap- 
Dagli studi sull'attività di law making nel Congresso statunitense sappiamo che: 1) l'equilibrio decisionale è indotto dall'assetto organizzativo e dalla divisione del lavoro all'interno del parlamento; 2) l'opacità del processo decisionale favorisce comportamenti di voto sofisticati e quindi lo scambio di voti; 3 ) la frequenza di maggioranze prossime all'unanimità dipende dalla lunghezza degli orizzonti temporali dei parlamentari, dall'incertezza sulla composizione delle coalizioni vincenti, e dal costo elettorale di un'eventuale esclusione da queste ultime o, al contrario, dai vantaggi di una partecipazione.

Il sistema elettorale, il sistema delle commissioni, le procedure di presentazione e votazione delle proposte di legge inducono congiuntamente i singoli parlamentari alla cooperazione. Nonostante le istituzioni parlamentari e il sistema politico italiano nel suo complesso fossero (e siano) almeno parzialmente diversi dalle istituzioni parlamentari e dal sistema politico statunitense, il loro funzionamento congiunto rendeva altrettanto probabile l'esito cooperativo.

Il parlamento italiano è organizzato in commissioni permanenti, la cui effettiva membership in passato era limitata dalla frammentazione partitica. Contrariamente alle commissioni della Camera dei Rappresentanti le commissioni del parlamento italiano possono, a determinate condizioni, legiferare. $\mathrm{Da}$ un lato, per conservare questa capacità è necessaria una unità di intenti ${ }^{26}$ probabilmente maggiore di quella richiesta ad una commissione della Camera dei Rappresentanti per ottenere dalla giunta del regolamento una regola di votazione favorevole in aula (Oleszek 1989); dall'altro, la ricompensa è pure maggiore: il completo controllo sul processo legislativo, per lo meno su quello relativo alla camera di appartenenza.

E questo potere è stato ampiamente utilizzato dalle commissioni stesse: fino alla $\mathrm{X}$ legislatura la stragrande maggioranza dei disegni di legge sono stati approvati in sede legislativa dalle commissioni, pressoché all'unanimità. Le regole di votazione sugli emendamenti impediscono, anche nel caso italiano, la riproposizione surrettizia di proposte già battute, garantendo il processo decisionale contro il caos. Quello che avviene nelle

26 È sufficiente infatti che un quinto dei componenti una commissione sia contrario all'assegnazione in sede legislativa perché il disegno di legge venga assegnato in sede referente. In tal caso nella migliore delle ipotesi il disegno di legge passerà all'esame finale dell'aula. 
commissioni è inoltre scarsamente visibile alla pubblica opinione (se non a quei settori direttamente beneficiari dei provvedimenti) e induce i singoli parlamentari a comportamenti strategici e allo scambio di voti.

L'arena elettorale di ciascun deputato era invece radicalmente diversa da quella statunitense. Il singolo deputato competeva per il voto di preferenza all'interno di un'ampia circoscrizione, e nulla lo garantiva a priori contro l'eventualità di sedere in commissione accanto ad un pericoloso concorrente presso il medesimo collegio, eventualità che un sistema elettorale uninominale rende impossibile. Al limite, proprio il meccanismo di assegnazione alle commissioni sulla base degli interessi maggiormente diffusi sul territorio della circoscrizione potrebbe in astratto rendere probabile quest'eventualità. Tuttavia, la divisione dell'elettorato in famiglie politiche fra loro contrapposte e la sua scarsa mobilità, insieme premessa ed effetto del sistema elettorale (ed alimentata dal fallimento del gioco delle grandi politiche), ridisegnava, all'interno di ogni circoscrizione, collegi virtuali più ridotti, ciascuno dei quali poteva avere in parlamento un unico rappresentante. I pericoli per il singolo parlamentare provenivano dagli sfidanti nelle file del medesimo partito, piuttosto che dai colleghi parlamentari di partiti avversi.

Paradossalmente, la natura ideologica della competizione partitica consentiva il particolarismo e l'accordo: non perché le leadership partitiche disattendessero la promessa di intransigenza e fermezza fatta in campagna elettorale, ma perché il contenuto di quella stessa promessa rendeva indirettamente $i$ singoli parlamentari, e soprattutto le seconde file, certi di detenere il monopolio della rappresentanza della propria constituency. Il sistema delle commissioni consentiva ai singoli parlamentari, rappresentanti di constituencies «simili», ma imprenditori politici su mercati non comunicanti, di accordarsi. E questo certo non contraddiceva la vocazione a rappresentare l'elettorato del proprio partito. Era infatti all'interno di quell'elettorato che ciascuno ritagliava i confini della propria constituency ${ }^{27}$.

27 Una caratteristica che esasperava il particolarismo ben oltre l'esempio americano era la preferenza multipla. I candidati del partito che fu di maggioranza relativa, attraverso sapienti alleanze, potevano essere eletti anche se la base autonoma di consenso era assai inferiore a quella che avrebbe consentito l'elezione. Una volta eletti, la constituency artificiosamente gonfiata dallo scambio delle preferenze tornava, nella fase della rappresentanza, alle sue dimensioni reali. Il parlamentare era indotto da un lato a proporre provvedimenti quasi ad personam, dall'altro a «sperimentare» l'introduzione di 
La stessa centralità del partito come elemento cognitivo nella scelta elettorale, come formidabile congegno di orientamento e stabilizzazione delle credenze, rendeva un ipotetico scontro condotto nelle commissioni da singoli parlamentari, o gruppi di parlamentari dello stesso partito, una forma di rassicurazione simbolica poco redditizia. L'elettore era infatti già ampiamente rassicurato dalle dichiarazioni giornaliere dei vertici partitici e, se mai avesse prestato attenzione ai lavori legislativi, lo avrebbe fatto nella speranza di ottenere il tal o tal altro provvedimento, e non un duello di retorica ed una gara di buone intenzioni.

La riflessione sul caso italiano ci ha indotto a considerare gli effetti simultanei di due diverse partite condotte, rispettivamente, dal partito (o meglio ancora dai suoi leader) e dal singolo parlamentare. Le regole rilevanti in ciascuna delle arene competitive in cui partito e parlamentare si trovano ad operare fanno sì che $\mathrm{i}$ giochi abbiano forme diverse, ma nello stesso tempo che $\mathrm{i}$ risultati ottenibili in ciascuno dei due tavoli possano influenzare la partita condotta nell'altro. Non solo l'assenza di decisioni nel gioco delle grandi politiche ha in parte o completamente il valore delle microdecisioni, ma il valore di una decisione nel gioco delle grandi politiche diventa in parte o completamente il valore assunto dallo stallo decisionale nel gioco delle leggine ${ }^{28}$. L'idea è che, sebbene i due giochi siano condotti da due tipi di attori diversi, ad un determinato esito di ciascun gioco (lo stallo decisionale), corrisponda la soluzione dell'altro. L'ipotesi implicita a questa raffigurazione è che in misura variabile l'insieme di attività connesse ai due giochi insista su un medesimo insieme di strutture (quelle appunto parlamentari). Tale circostanza rende $\mathrm{i}$ due giochi in competizione fra loro.

La distribuzione dei pay-offs in ciascun gioco e la capacità di ciascun gioco di influenzare la distribuzione dei pay-offs di quello contiguo sono il risultato delle regole e circostanze rilevanti di cui parlamentari e leader di partito devono tener conto. Così, nel caso italiano la distanza fra le posizioni ideologiche dei partiti, e quindi i danni che ciascuna delle parti riteneva di essere destina-

proposte di legge dal contenuto assai vario nel tentativo di ampliare i confini della propria constituency.

28 Abbiamo chiamato questo modello «gioco intrecciato» sebbene le definizioni dei primi autori che si sono occupati di giochi intrecciati (linked games) escludano da quella categoria il caso di due coppie di giocatori diversi che giocano due giochi in cui le distribuzioni dei pay-offs (e quindi le soluzioni) sono interdipendenti. Vedi Alt e Eichengreen (1989); e Alt, Putnam e Shepsle (1988). 
ta a subire in caso di «inganno» della parte antagonista, erano sufficientemente elevati rispetto ai benefici prodotti dagli accordi fra parlamentari su materie non controverse ovvero rispetto ai benefici dello stallo decisionale sui grandi temi (vedi caselle DC e CD della figura 1a e casella CC della figura $1 b$ ).

Ciascuna caratteristica politico-istituzionale in una rappresentazione in forma di gioco intrecciato perde un suo peso assoluto per acquisirne uno relativo. Così un processo di depolarizzazione $^{29}$ (fig. 2a), rappresentato da un avvicinamento dei valori dei pay-offs dei principali partiti nel caso di decisioni favorevoli ad uno solo fra loro, dovrebbe condurre ad una maggiore propensione a decidere «grandi politiche» sia direttamente, sia attraverso la diminuita segmentazione dei mercati partitici che accompagnerebbe un avvicinamento delle posizioni dei partiti. In quest'ultimo caso, la maggiore mobilità interpartitica renderebbe molto meno munite e impermeabili le singole constituencies dei parlamentari rispetto alla concorrenza di altri parlamentari della medesima circoscrizione, e, quindi, deprezzerebbe gli accordi in commissione ed il valore di uno stallo decisionale nel gioco delle grandi politiche (vedi i pay-offs in DD della figura 2a).

Se tuttavia una riforma elettorale ridefinisce l'ambiente competitivo dei parlamentari in termini di collegi uninominali, magari proprio con l'intento di accelerare il processo di depolarizzazione, allora, ferma restando l'organizzazione per commissioni all'interno del parlamento, il valore dei microaccordi aumenta ulteriormente (Fiorina 1981; Weingast 1979). Tale aumento potrebbe vanificare il processo di depolarizzazione e far preferire lo stallo decisionale nel gioco delle grandi politiche ${ }^{30}$ (fig. 2b). Il valore dei microaccordi è infatti destinato ad aumentare perché il singolo rappresentante è più facilmente riconoscibile da parte degli interessi radicati all'interno del proprio collegio e d'altra parte questa maggiore riconoscibilità consente la creazione di un patrimonio di consensi parzialmente indipendente dalle strategie del partito. Esiste pertanto una maggiore probabilità di essere puniti o premiati sulla base dei risultati conseguiti durante l'attività legislativa e quindi una maggiore propensione a cooperare con gli altri parlamentari della medesi-

29 In un processo di depolarizzazione le soluzioni DC e CD sono entrambe preferibili all'assenza di decisioni. L'ordine degli esiti per i singoli diventa DC > CC > CD > DD.

30 La trattazione si basa sull'assunto che nel frattempo sia le regole interne al parlamento che il rapporto governo-parlamento non mutino. 


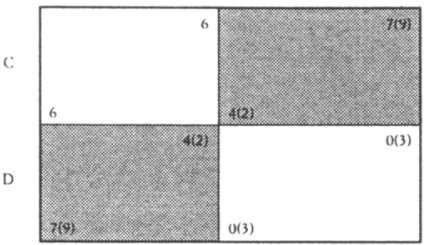

D

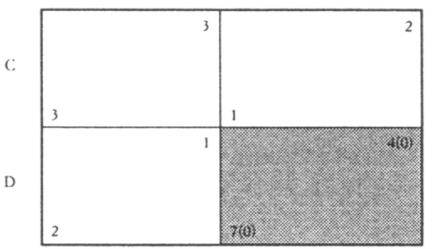

\section{Soluzione del gioco}

$(\ldots)=$ Valori nel gioco di fig. 1

FIG. 2a. Processo di depolarizzazione.

ma commissione, che in virtù del sistema elettorale non possono essere mai diretti concorrenti.

In altri termini la depolarizzazione ha un valore relativo. D'altra parte, anche i mutamenti nella distribuzione delle poste del gioco delle leggine possono modificare le soluzioni del gioco delle grandi politiche. Un ricambio massiccio della classe parlamentare ed una diffusa incertezza ${ }^{31}$ dei nuovi legislatori nella percezione degli interessi delle proprie constituencies possono rendere difficile e poco redditizio raggiungere compromessi: ciascun legislatore non conosce le preferenze di policy degli altri membri della commissione, conoscendo, per altro, poco bene perfino quelle della propria constituency. Una simile situazione potrebbe spingere $i$ parlamentari ad adottare per intero le priorità «politiche» ufficiali del partito: in tal caso lo spazio politico diverrebbe unidimensionale e non esisterebbero neppure i presupposti dello scambio. In ogni caso lo stallo decisionale sulle «grandi questioni» cessa di essere la soluzione

31 Per alcuni suggerimenti sul rapporto fra i lavori nelle commissioni e incertezza politica vedi Fiorina (1987). 
Gioco delle leggine

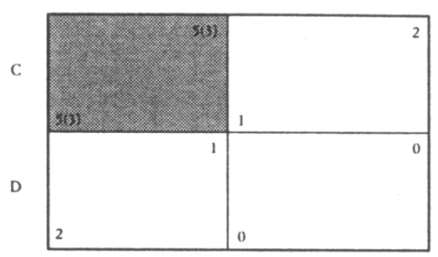

Gioco delle grandi politiche

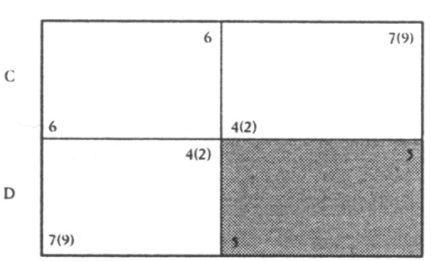

Soluzione del gioco

(...) = Valori nel gioco di fig 1

FIG. 2b. Processo di depolarizzazione con collegi uninominali.

del gioco perché nel frattempo si sospende il gioco delle leggine, ovvero si perviene ad una soluzione $\mathrm{DD}$ o CD o DC.

Per leggere adeguatamente la storia appena narrata sulle matrici dei due giochi possiamo scomporla in due stadi. In un primo stadio il valore diminuito della cooperazione congiunta nel gioco delle leggine (da 5,5 a 1,1) è tale da spingere a soluzioni diverse dalla cooperazione congiunta nel gioco delle leggine. Quali che siano le soluzioni del gioco delle leggine (defezione reciproca inclusa) esse non producono più pay-offs abbastanza elevati perché la defezione congiunta nel gioco delle grandi politiche resti una soluzione. Gli attori partitici nelle mutate circostanze preferiscono comunque raggiungere una qualche decisione, accettando anche che quest'ultima sia più vantaggiosa per la controparte. La soluzione del gioco delle grandi politiche diventa allora $(9,2)$ o $(2,9)$. In un secondo stadio tale soluzione diventa pure il valore della defezione congiunta nel gioco delle leggine. A sua volta il valore della defezione congiunta nel gioco delle grandi politiche, che ora corrisponde ad un'analoga as- 
Gioco delle leggine

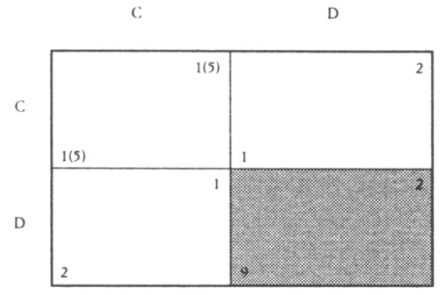

Gioco delle grandi politiche

D

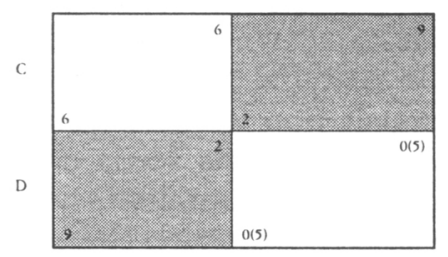

FIG. 3. Ricambio della classe politica.

senza di decisioni nel gioco delle leggine, si azzera completamente (ed ecco perché DD nelle grandi politiche diventa 0 ).

\section{Conclusioni provvisorie}

In questo breve saggio mi sono proposto di offrire un'interpretazione dell'attività legislativa del parlamento italiano della Prima Repubblica alternativa alle spiegazioni fino ad oggi fornite, ma allo stesso tempo composta dalle parti, a mio parere più convincenti, di quelle stesse spiegazioni. Nel far questo mi sono posto i medesimi problemi che sono al centro della ricerca di impostazione razional-deduttiva sul Congresso americano ed ho utilizzato un modello di gioco intrecciato. Questa scelta porta con sé alcune conseguenze sull'annosa questione riguardante il ruolo dei partiti nei processi decisionali.

Nel dibattito internazionale ed ormai anche in quello italiano l'importanza dei partiti nell'attività di policy making è stata messa in dubbio ${ }^{32}$. Lo stesso bersaglio polemico da cui muove

32 Vedi Cotta (1994) e Giuliani (1995). 
questo contributo è, appunto, l'importanza malposta in passato sul sistema dei partiti per spiegare l'attività legislativa in parlamento. E tuttavia, sebbene nella mia interpretazione i protagonisti del consenso non siano i partiti, ma piuttosto i singoli parlamentari, meglio ancora se di secondo piano, e sebbene trovi importante lo studio delle commissioni parlamentari quali veri e propri soggetti politici, non si può certo inferire dal mio argomento che qualsiasi sistema di partito e qualsiasi sistema elettorale lascino immutati i processi legislativi in parlamento. L'indipendenza del contenuto delle decisioni da programmi o direttive degli organi di partito o da una contrattazione realmente «partitica» non prova l'assenza di un'influenza del sistema di interazione partitica-elettorale sulle logiche di azione del singolo parlamentare. L'ampio margine di manovra del singolo in determinate aree decisionali non avviene nonostante il forte richiamo ideologico del partito sulla scena elettorale ma al contrario, dato un certo sistema elettorale, in virtù di quello. Così come è altrettanto possibile sostenere che alcune decisioni nel gioco delle grandi politiche, prese a maggioranza, non avvengono solo per un mutamento dell'ambiente competitivo elettorale dei partiti ma pure per mutamenti all'interno dell'ambiente di contrattazione dei singoli legislatori in parlamento. Il legame che la teoria democratica postula fra il momento del voto e quello delle decisioni parlamentari non è, nella mia interpretazione, completamente rescisso: semmai la pluralità degli attori coinvolti, il funzionamento congiunto delle diverse regole che si frappongono fra l'uno e l'altro momento, rendono la ricostruzione di tale legame tortuosa e difficile. E, cosa ancora più importante, l'affermazione di tale legame va di pari passo con la negazione di una qualche priorità causale di uno dei due momenti sull'altro. Come scrivono Cain, Ferejohn e Fiorina alla fine di Personal Vote (1987, 216-217): «Né il voto personale, né le regole parlamentari sono invariabilmente la causa originaria; semmai, sono entrambi endogeni come lo sono le istituzioni legislative, la natura del processo politico (di policy) e lo stesso sistema di partito. Provate a cambiare uno di questi elementi e gli altri tenderanno ad aggiustarsi di conseguenza». 
APPENDICE

Intreccio principale

Gioco delle grandi politiche

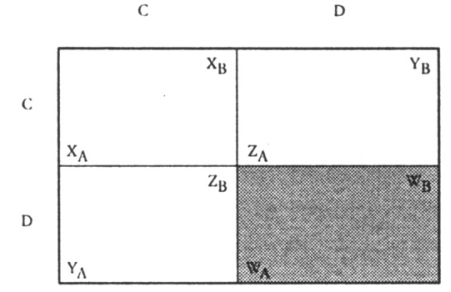

Soluzione del gioco

Per il giocatore $A$ (e simmetricamente per il giocatore $B$ )

$\mathrm{YA}>\mathrm{XA}>\mathrm{WA}>\mathrm{ZA}$; la soluzione del gioco è (WA,WB)

Gioco delle leggine
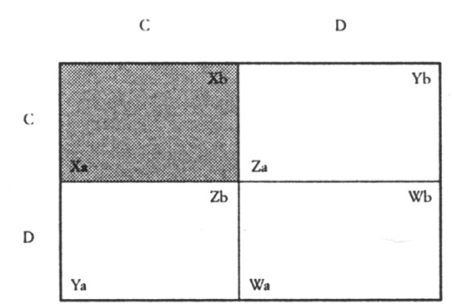

Per il giocatore a (e simmetricamente per il giocatore b)

$\mathrm{Xa}>\mathrm{Ya}>\mathrm{Za}>\mathrm{Wa}$; la soluzione del gioco è $(\mathrm{Xa}, \mathrm{Xb})$

Le condizioni dell'intreccio sono

$W A=\left\{\begin{array}{l}\text { per Soluzione gioco leggine }(\mathrm{Wa}, \mathrm{Wb}): 0 \\ \text { per Soluzione gioco leggine }(\mathrm{Xa}, \mathrm{Xb}): \mathrm{s}(\mathrm{Xa})\end{array}\right.$

$(\mathrm{Ya}, \mathrm{Zb}): \mathrm{s}(\mathrm{Ya})$;

$(\mathrm{Za}, \mathrm{Yb}): \mathrm{s}(\mathrm{Za}) \quad 0<\mathrm{s}<1$ 
$\mathrm{Wa}=\left\{\begin{array}{l}\text { per Sol. gioco grandi politiche }(\mathrm{WA}, \mathrm{WB}): 0 \\ \text { per Sol. gioco grandi politiche }(\mathrm{XA}, \mathrm{XB}): \mathrm{S}(\mathrm{XA})\end{array}\right.$

$(Y A, Z B): S(Y A)$

$(\mathrm{ZA}, \mathrm{YB}): \mathrm{S}(\mathrm{ZA}) \quad 0<\mathrm{S}<1$

Per le condizioni accennate nel testo sembra plausibile assumere $s>\mathrm{S}$, ossia che l'impatto di una decisione presa nel gioco delle leggine sui pay-offs della reciproca defezione nel gioco delle grandi politiche sia maggiore dell'impatto di una decisione presa nel gioco delle grandi politiche sulla reciproca defezione nel gioco delle leggine. In altre parole il consenso conquistato e mantenuto dal singolo parlamentare attraverso la contrattazione minuta in parlamento si riversa senza eccessive perdite sul partito in quanto tale. Viceversa, gli eventuali benefici di una decisione nel gioco delle grandi politiche presa dalle leadership partitiche verranno egualmente ripartiti a favore di tutti i parlamentari dello stesso partito e quindi a favore anche di eventuali concorrenti diretti in carica, se non addirittura a favore di potenziali candidati dello stesso partito nella circoscrizione di provenienza.

Processo di depolarizzazione

Depolarizzazione moderata

(1) Se nel gioco delle grandi politiche

$\mathrm{YA}>\mathrm{XA}>\mathrm{ZA}>\mathrm{WA}$

e $\mathrm{YB}>\mathrm{XB}>\mathrm{ZB}>\mathrm{WB}$

la soluzione del gioco è $(\mathrm{YA}, \mathrm{ZB})$ o $(\mathrm{YB}, \mathrm{ZA})$

(2) Se nel gioco delle leggine

$\mathrm{Xa}>\mathrm{S}(\mathrm{YA})>\mathrm{Ya}>\mathrm{Za}$

$\mathrm{Xb}>\mathrm{S}(\mathrm{ZB})>\mathrm{Yb}>\mathrm{Zb}$

la soluzione del gioco delle leggine resta $(\mathrm{Xa}, \mathrm{Xb})$

(3) Poiché nel gioco delle grandi politiche WA e WB non sono cambiati e sono equivalenti rispettivamente a $\mathrm{s}(\mathrm{Xa})$ e $\mathrm{s}(\mathrm{Xb})$ la so- 
luzione del gioco delle grandi politiche resta $(\mathrm{YA}, \mathrm{ZB})$ o (YB, ZA)

Gioco delle grandi politiche

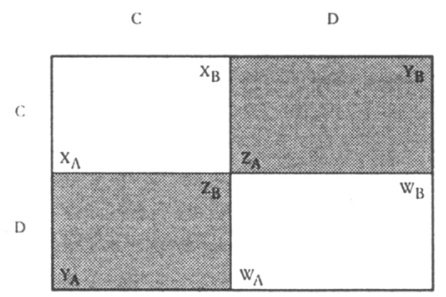

Gioco delle leggine

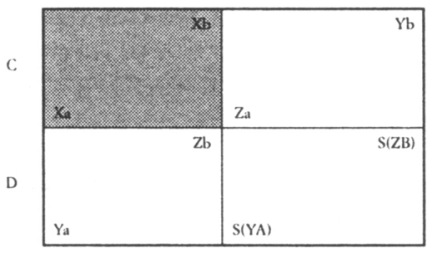

Ossia un moderato processo di depolarizzazione senza una diminuzione dei pay-offs corrispondenti all'accordo nel gioco delle leggine - dovuta per esempio ad una minor segmentazione del mercato partitico - consente la compresenza di decisioni in entrambi i giochi.

Depolarizzazione forte

(1) Se nel gioco delle leggine $\mathrm{S}(\mathrm{YA})=\mathrm{Wa}>\mathrm{X} \mathrm{a}>\mathrm{Ya}>\mathrm{Za}$ $\mathrm{S}(\mathrm{ZB})=\mathrm{Wb}>\mathrm{Xb}>\mathrm{Yb}>\mathrm{Zb}$ la soluzione del gioco delle leggine è allora $\mathrm{S}(\mathrm{YA}), \mathrm{S}(\mathrm{ZB})$ ovvero nessuna decisione

(2) Poiché $\mathrm{WA}=\mathrm{WB}=\mathrm{S}(\mathrm{Wb})=\mathrm{S}(\mathrm{Wa})=0$ 
la soluzione del gioco delle grandi politiche è ancora (YA, ZB) o (YB, ZA)

Gioco delle grandi politiche

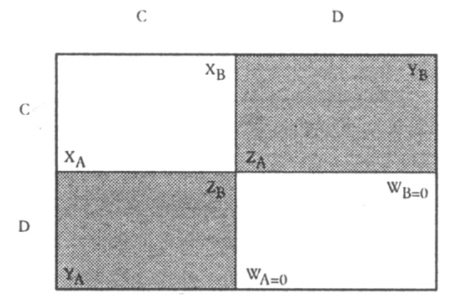

Gioco delle leggine

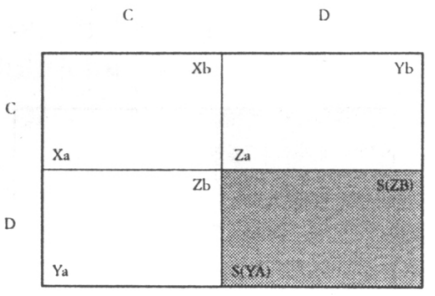

Ossia un forte processo di depolarizzazione - accompagnato magari da una diminuzione dei pay-offs corrispondenti all'accordo nel gioco delle leggine - consente decisioni solo nel gioco delle grandi politiche.

Processo di depolarizzazione con collegi uninominali

(1) Se nel gioco delle grandi politiche

$\mathrm{YA}>\mathrm{XA}>\mathrm{ZA}>\mathrm{WA}$

e $\mathrm{YB}>\mathrm{XB}>\mathrm{ZB}>\mathrm{WB}$

la soluzione del gioco è (YA, ZB) o (YB, ZA)

(2) Se nel gioco delle leggine in virtù dei collegi uninominali i valori di $\mathrm{Xa}$ e $\mathrm{Xb}$ aumentano ulteriormente

$\mathrm{Xa}>\mathrm{S}(\mathrm{YA})>\mathrm{Ya}>\mathrm{Za}$ 
$\mathrm{Xb}>\mathrm{S}(\mathrm{ZB})>\mathrm{Yb}>\mathrm{Zb}$

la soluzione del gioco delle leggine resta $(\mathrm{Xa}, \mathrm{Xb})$

(3) Poiché nel gioco delle grandi politiche WA e WB sono cambiati e poiché equivalenti rispettivamente a $s(\mathrm{Xa})$ e $s(\mathrm{Xb})$ sono aumentati

allora se WA $>\mathrm{ZA}>=\mathrm{XA}$ e $\mathrm{WB}>\mathrm{Zb}>=\mathrm{XB}$ la soluzione del gioco diventa WA, WB

e nel gioco delle leggine $\mathrm{Wa}=\mathrm{Wb}=0$

Gioco delle grandi politiche

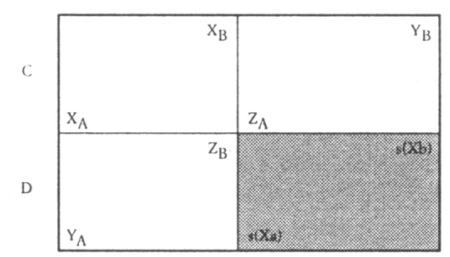

\section{Gioco delle leggine}
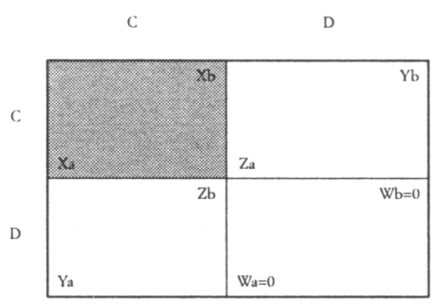

Ricambio della classe politica senza depolarizzazione

(1) Se nel gioco delle leggine $\mathrm{Ya}>\mathrm{Xa}$ e $\mathrm{Zb}>\mathrm{Wb}$ o $\mathrm{Yb}>\mathrm{Xb}$ e $\mathrm{Za}>\mathrm{Wa}$ allora la soluzione del gioco delle leggine è $\mathrm{Ya}, \mathrm{Zb}$ o $\mathrm{Yb}, \mathrm{Za}$

(2) Se nel gioco delle grandi politiche $\mathrm{WA}=\mathrm{S}(\mathrm{Ya})<\mathrm{YA}$ o $\mathrm{WB}=\mathrm{S}(\mathrm{Yb})<\mathrm{YB}$ allora la soluzione nel gioco delle grandi politiche diventa YA, $\mathrm{ZB}$ o $\mathrm{YB}, \mathrm{ZA}$ 
(3) Poiché nel gioco delle leggine $\mathrm{Wa}=s(Y A)$ e $\mathrm{Wb}=s(\mathrm{ZB})$ nel caso di YA, ZB come soluzione del gioco delle grandi politiche e $\mathrm{Wb}=\mathrm{s}(\mathrm{YB})$ e $\mathrm{Wa}=\mathrm{s}(\mathrm{ZA})$ nel caso di $\mathrm{YB}, \mathrm{ZA}$ come soluzione del gioco delle grandi politiche

Se $\mathrm{Wa}>\mathrm{Za}$ e $\mathrm{Wb}>\mathrm{Zb}$ allora la soluzione del gioco delle leggine diventa $\mathrm{Wa}, \mathrm{Wb}$

Gioco delle grandi politiche

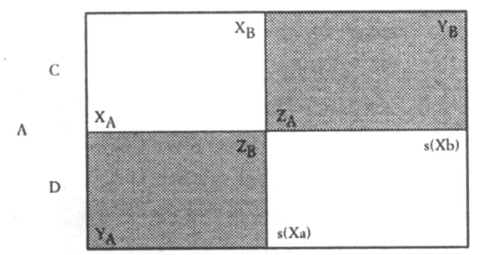

Gioco delle leggine

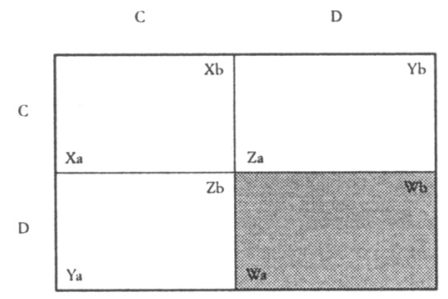

\section{Riferimenti bibliografici}

Almond, G.A. (1956), Comparative Political Systems, in «Journal of Politics», vol. 18, pp. 391-409.

Alt, J.E., R.D. Putnam e K.A. Shepsle (1988), The Architecture of Linkage, mimeo.

Alt, J.E. e B. Eichengreen (1989), Parallel and Overlapping Games: Theory and an Application to the European Gas Trade, in «Economics and Politics», 2, pp. 119-144.

Arrow, K.J. (1951, 1963), Social Choice and Individual Values, New York-New Haven, Yale University Press.

Black, D. (1958), The Theory of Committees and Elections, London, Cambridge University Press. 
Brady, D.W. (1988), Critical Elections and Congressional Policy Making, Stanford, Stanford University Press.

Caciagli, M., F. Cazzola, L. Morlino e S. Passigli (a cura di) (1994), L'Italia fra crisi e transizione, Roma-Bari, Laterza.

Cain, B., J. Ferejohn e M. Fiorina (1987), The Personal Vote: Constituency Service and Electoral Independence, Cambridge (Mass.), Harvard University Press.

Cavazza, F.L. e S.R. Graubard (1974), Il caso italiano, Milano, Garzanti.

Cazzola, F. (1972), Consenso e opposizione nel parlamento italiano. Il ruolo del PCI dalla I alla IV legislatura, in «Rivista Italiana di Scienza Politica», vol. 2, pp. 171-196.

Chubb, J.E. e P.E. Peterson (a cura di) (1989), Can the Government Govern?, Washington D.C., The Brookings Institutions.

Copeland, G. e S. Patterson (a cura di) (1994), Parlaments in the Modern World. Changing Institutions, Ann Arbor, The University of Michigan Press.

Cotta, M. (1994a), Il governo di partito in Italia, in Caciagli, Cazzola, Morlino e Passigli, pp. 119-139.

- (1994b), The Rise and Fall of the Centrality of the Italian Parliament. Transformation of Executive-legislative Subsystem after the Second World War, in Copeland e Patterson, pp. 59-84.

- (1996), La crisi del governo di partito all'italiana, in Cotta e Isernia, pp. 11-52.

Cotta, M. e P. Isernia (a cura di) (1996), Il gigante dai piedi di argilla. La crisi del regime partitocratico in Italia, Bologna, Il Mulino.

Cox, G.W e M.D. McCubbins (1993), Legislative Leviathan. Party Government in the House, Berkeley, University of California Press.

Crecine, J.P. (a cura di) (1981), Research in Public Policy Analysis and Management, Greenwich, Jai Press.

De Micheli, C. (1994), Crisi del Policentrismo? Alcuni aspetti del funzionamento del parlamento italiano dal 1983 al 1992, Relazione presentata al convegno annuale SISP.

- (1997), L'attività legislativa dei governi al tramonto della prima repubblica, in «Rivista Italiana di Scienza Politica», vol. 27, pp. 149. 187.

Denzau, A., W. Riker e K. Shepsle (1985), Farqubarson and Fenno: Sophisticated Voting and Home Style, in «American Political Science Review», vol. 79, pp. 1117-1135.

Di Palma, G. (1978), Sopravvivere senza governare. I partiti nel Parlamento italiano, Bologna, Il Mulino.

- (1987), Parlamento-arena o parlamento di trasformazione?, in «Rivista Italiana di Scienza Politica», vol. 17, pp.179-202.

Fabbrini, S. (1994), Quale democrazia. L'Italia e gli altri, Bari, Laterza. Fiorina, M. (1981), Universalism, Reciprocity, and Distributive Policymaking in Majority Rule Institutions, in Crecine, pp. 197-221. 
- (1987), Alternative Rationales for Restrictive Procedures, in «Journal of Law, Economics, and Organization», 3, pp. 337-343.

Giuliani, M. (1995), Le politiche pubbliche fra sistemi di partito e policy networks, in G. Regonini (a cura di), Politiche pubbliche e democrazia, Napoli, Esi, pp. 219-240.

- (1996), Measures of Consensual Law-making: the Italian «Consociativismo», Quaderno di Ricerca Poleis, n. 12.

Krehbiel, K. (1987), Sophisticated Committees and Structure Induced Equilibria in Congress, in McCubbins e Sullivan, pp. 376-402.

- (1988), Spatial Models of Legislative Choice, in «Legislative Studies Quaterly», vol. 13, pp. 259-321.

- (1991), Information and Legislative Organization, Ann Arbor, The University of Michigan Press.

- (1993), Where's the Party? in «British Journal of Political Science», vol. 23, pp. 235-266.

Lijphart, A. (1968), Typologies of Democratic Systems, in «Comparative Political Studies», vol. 1, pp. 3-44.

- (1984), Democracies. Patterns of Majoritarian and Consensus Government in Twenty-One Countries, New Haven, Yale University Press; trad. it. Le Democrazie Contemporanee, Bologna, Il Mulino, 1988.

Mayhew, D. (1974), Congress: The Electoral Connection, New Haven, Yale University Press.

McCubbins, M. e T. Sullivan (a cura di) (1987), Congress: Structure and Policy, Cambridge-New York, Cambridge University Press.

McKelvey, R. (1976), Intransitivities in Multidimensional Voting Models with Some Implications for Agenda Control, in «Journal of Economic Theory», vol. 12, pp. 472-482.

Morisi, M. (1992), Le leggi del consenso. Partiti e interessi nei primi parlamenti della Repubblica, Soveria Mannelli, Rubbettino.

Oleszek, W. (1989), Congressional Procedure and the Policy Process, Washington, Congressional Quarterly ( ${ }^{a}$ ed.).

Pappalardo, A. (1979), Le condizioni della democrazia consociativa. Una critica logica ed empirica, in «Rivista Italiana di Scienza Politica», vol. 9, pp. 367-446.

Pizzorno, A. (1993), Le difficoltà del consociativismo, in A. Pizzorno, Le radici della politica assoluta, Milano, pp. 285-313.

Plott, C.R. (1967), The Notion of Equilibrium and its Possibility under Majority Rule, in «American Economic Review», vol. 57, pp. 787806.

Regonini, G. (1994), Partiti, reti, giochi, politiche pubbliche, in Caciagli, Cazzola, Morlino e Passigli, pp. 163-189.

- (1996), Partiti e pensioni: legami mancanti, in Cotta e Isernia, pp. 73-137.

Riker, W.H. (1962), The Theory of Political Coalitions, New Haven, Yale University Press. 
Rohde, D.W. e K.A. Shepsle (1973), Democratic Committee Assignments in the House of Representatives: Strategic Aspects of a Social Choice Process, in «American Political Science Review», vol. 67, pp. 889-905.

Sartori, G. (1963), Dove va il Parlamento in Italia, in Somogy, Lotti, Predieri e Sartori, pp. 281-386.

- (1974), Rivisitando il pluralismo polarizzato, in Cavazza e Graubard, pp. 296-323.

Scharpf, F. (1990), Games Real Actors Could Play: The Problem of Mutual Predictability, in «Rationality and Society», 2, pp. 471-494.

Schofield, N. (1978), Instability of Simple Dynamics Games, in «Review of Economic Studies», vol. 45, pp. 575-594.

Shepsle, K.A. (1978), The Giant Jigsaw Puzzle, Chicago, The University of Chicago Press.

- (1979a), The role of Institutional Structure in the Creation of Policy Equilibrium, Beverly Hills-London, Sage, pp. 249-281.

- (1979b), Institutional Arrangements and Equilibrium in Multidimensional Voting Models, in «American Journal of Political Science», vol. 23, pp. 27-60.

- (1989), The Changing Textbook of Congress, in Chubb e Peterson, pp. 238-266.

Shepsle, K.A. e B. Weingast (1981a), Political Preference for the Pork Barrel: A Generalization, in «American Journal of Political Science», vol. 25, pp. 96-111.

- (1981b), Structure-induced Equilibrium and Legislative Choice, in «Public Choice», vol. 37, pp. 503-519.

Somogyi, S., L. Lotti, A. Predieri e G. Sartori (1963), Il parlamento italiano: 1946-1963, Napoli, Esi.

Strom, G.S. (1990), The Logic of Lawmaking: A Spatial Theory Approach, Baltimore, The Johns Hopkins University Press.

Tsebelis, G. (1990), Nested Games. Rational Choice in Comparative Politics, Berkeley, University of California Press.

- (1995), Decision Making in Political Systems: Veto Players in Presidentialism, Parliamentarism, Multicameralism and Multipartyism, in «British Journal of Political Science», vol. 25, pp. 289-325.

Tullock, G. (1981), Why so much stability?, in «Public Choice», vol. 37, pp. 189-202.

- (1984), La motivazione del voto, in S. Carrubba e D. Da Empoli (a cura di), Scelte pubbliche, Firenze, Le Monnier, pp. 37-97.

Weingast, B. (1979), A Rational Choice Perspective on Congressional Norms, in «American Journal of Political Science», vol. 23, pp. 245-262.

Weingast, B. e W. Marshall (1988), The Industrial Organization of Congress, in «Journal of Political Economy», vol. 96, pp. 132-163.

Zucchini, F. (1995), Giochi della rappresentanza e parlamento negli Stati Uniti ed in Italia, Tesi di dottorato. 